\title{
On the Intersections of Longest Cycles in a Graph
}

\author{
lain A. Stewart and Ben Thompson
}

\section{CONTENTS}

1. Introduction

2. The Basic Strategy

3. Remarks on the Computations

4. The Different Cases

References

Electronic Availability
We confirm a conjecture, due to Grötschel, regarding the intersection vertices of two longest cycles in a graph. In particular, we show that if $G$ is a graph of circumference at least $k+1$, where $k \in\{6,7\}$, and $G$ has two longest cycles meeting in a set $W$ of $k$ vertices, then $W$ is an articulation set. Grötschel had previously proved this result for $k \in\{3,4,5\}$ and shown that it fails for $k>7$. As corollaries, we obtain results regarding the minimum lengths of longest cycles in certain vertex-transitive graphs. Our proofs are novel in that they make extensive use of a computer, although the programs themselves are straightforward.

\section{INTRODUCTION}

We consider the intersection vertices of two longest cycles in a graph. (A cycle is a closed path with no repeated vertices.) As remarked in [Grötschel 1984], a considerable amount of work has been done regarding the length of longest cycles in various graphs, but not much attention has been paid to how these longest cycles intersect. The present article confirms a conjecture posed in [Grötschel 1984], and can be regarded as a continuation of that paper (which is the one we will have in mind whenever we refer to Grötschel's work below).

Grötschel considered graphs with two longest cycles meeting in up to five vertices. His main result was the following.

Theorem 1.1 [Grötschel 1984]. Let $k \in\{1,2,3,4,5\}$, and let $G$ be a graph with at least $k+1$ vertices. Suppose that $G$ has two longest cycles meeting in a set $W$ of exactly $k$ vertices. Then $W$ is an articulation set of $G$.

Saying that $W$ is an articulation set means that, if we remove from $G$ all vertices in $W$ and all edges incident on them, $G$ becomes disconnected. 
One could, of course, ask what is the largest number $k$ for which Theorem 1.1 holds. However, Grötschel produced a graph with more than 7 vertices and two longest cycles of length 6 meeting in a set $W$ of exactly 6 vertices, where $W$ is not an articulation set: hence, this largest number is 5 .

Grötschel shows in the proof of Theorem 1.1 that the case when the longest cycles have length $k$ is straightforward, and that all interesting situations arise when we assume that they have length at least $k+1$ (that is, that $G$ has circumference at least $k+1$ ). Grötschel also notes that the Petersen graph $P$ is a graph with 10 vertices where each longest cycle is of length 9 and, moreover, $P$ has two longest cycles meeting in a set $W$ of exactly 8 vertices that is not an articulation set. Hence, the largest number for which a restricted version of Theorem 1.1 to graphs of circumference at least $k+1$ might hold is certainly less than 8 .

Grötschel conjectured that this restricted version is true for $k=6$ and $k=7$. We confirm this conjecture. More precisely, we prove the following.

Theorem 1.2. Let $k \in\{6,7\}$ and let $G$ be a graph whose circumference is at least $k+1$. Suppose that $C$ and $D$ are distinct longest cycles of $G$ meeting in a set $W$ of exactly $k$ vertices. Then $W$ is an articulation set of $G$.

The proof Grötschel gave of Theorem 1.1 consisted in studying each different way in which the two longest cycles can intersect. As one might expect, as $k$ increases, the number of cases to study rises fast. When $k=5$ there are 4 different cases; however, when $k=6$ there are 10 , and when $k=7$ there are 25. Therefore extrapolating this method for $k>5$ is impractical.

As we show, much of the labour can be removed from the problem by employing a computer: a lot of the cases to be considered melt away after the computer analysis, and the remainder can easily be resolved using the computational results and some simple reasoning. Computer results may be treated with scepticism by some, but in this case the program is very simple to implement, and the result can be easily duplicated by the reader. See also the section on Electronic Availability at the end of the article.

Before proceeding with the proof of Theorem 1.2, we give an application of that result to the theory of vertex-transitive graphs, as Grötschel did with Theorem 1.1 (a graph is vertex-transitive if its automorphism group acts transitively on the set of vertices). The fact that in Theorem 1.2 the graph $G$ has circumference at least $k+1$ means that our applications do not mirror Grötschel's exactly.

Babai [1979] showed that every $k$-connected vertex-transitive graph $G$ with $n \geq 4$ vertices has a cycle of length greater than $(3 n)^{1 / 2}$. (A graph is $k$-connected if any articulation set has size at least $k$.) Grötschel essentially used this result and Theorem 1.1 to show that every $k$-connected vertex-transitive graph $G$, for $k \in\{3,4,5\}$, contains a cycle of length greater than $(k n)^{1 / 2}$. To obtain a similar result using Theorem 1.2, we need to be sure that our $k$-connected vertex-transitive graph, where $k \in\{6,7\}$, has circumference at least $k+1$. However, Babai's result, quoted above, ensures that this is the case when $k=6$ and $n \geq 12$, and when $k=7$ and $n \geq 17$. Theorem 1.2 now implies that, if $k=6$ and $n \geq 12$ or if $k=7$ and $n \geq 17$, two longest cycles in a $k$-connected vertextransitive graph $G$ (with $n$ vertices) intersect in at least $k$ vertices. An easy modification of Babai's proof now yields:

Corollary 1.3. Let $G$ be a $k$-connected vertex-transitive graph with $n$ vertices. If $k=6$ and $n \geq 12$, or if $k=7$ and $n \geq 17$, then $G$ has a cycle of length greater than $(k n)^{1 / 2}$.

Also, vertex-transitive graphs are regular. By a result of Mader [1971] and Watkins [1970], the connectivity of a connected $d$-regular graph is at least $\frac{2}{3}(d+1)$. Thus, using Corollary 1.3, we obtain:

Corollary 1.4. Let $G$ be a connected vertex-transitive graph with $n$ vertices. If $G$ is 8-regular and $n \geq 12$ then $G$ has a cycle of length greater than $(6 n)^{1 / 2}$, and if $G$ is 10-regular and $n \geq 17$ then $G$ has a cycle of length greater than $(7 n)^{1 / 2}$. 


\section{THE BASIC STRATEGY}

An undirected graph (hereafter, just a graph) is written as $G=(V, E)$, where $V$ denotes the set of vertices and $E$ the set of edges of $G$. Our graphs do not have multiple edges or self-loops, except when otherwise stated. A path in $G$ is a sequence of vertices such that each vertex is connected to its successor (if it has one) by a vertex. A closed path is defined likewise, with "successor" understood modulo the length of the sequence. A simple path is one on which every vertex appears at most once. A cycle is a closed simple path. The circumference of $G$ is the length of a longest cycle in $G$. A Hamiltonian cycle in $G$ is a cycle passing through every vertex of $V$. A graph is connected if any two vertices are joined by a path. An articulation set $W$ in a graph $G$ is a set of vertices whose removal from $G$ (along with any incident edges) causes the resulting graph to be disconnected. A graph is $k$ connected, for some $k>1$, if it has no articulation set of size less than $k$.

Let $G=(V, E)$ be a graph with circumference at least $k+1$, where $k \in\{6,7\}$, and suppose that $C$ and $D$ are two longest cycles of $G$ meeting in a set $W$ of $k$ vertices (that is, $C$ and $D$ have exactly the $k$ vertices of $W$ in common). Our aim is to prove Theorem 1.2. To do this we proceed as follows.

Let $H(G)$ be the subgraph of $G$ consisting of the two cycles $C$ and $D$. From this subgraph $H(G)$ construct another graph $X(G)$, possibly with multiple edges, where $X(G)$ has (a copy of) $W$ as its vertex set and there is an edge joining two vertices $x$ and $y$ of $X(G)$ if and only if there is a subpath of $C$ or a subpath of $D$ joining $x$ and $y$ in $H(G)$ on which there are no vertices of $W$ (apart from $x$ and $y)$. In $X(G)$ there are at most two edges joining any pair of vertices of $X(G)$. If there are two such edges, we say they are parallel. In short, the graph $X(G)$ consists of two cycles of length $k$ on the same set of $k$ vertices, intersecting in the same fashion as do the cycles $C$ and $D$ in $G$. Let $Y$ and $Z$ be the cycles of $X(G)$ corresponding to the cycles $C$ and $D$ of $G$.
The $H$-length of an edge $x y$ of $X(G)$ is the length of the corresponding subpath connecting $x$ and $y$ in $H(G)$ : we denote the $H$-length of the edge $x y$ by $|x y|$ (so $|x y|=|y x|$ ). By hypothesis, there is an edge $e$ of $Y$ and an edge $f$ of $Z$ whose $H$-lengths are greater than 1 . Hence, the subpaths of $C$ and $D$ corresponding to $e$ and $f$, say $\rho(e)$ and $\rho(f)$, each contain at least one vertex that is not in $W$. If there is a path in $G$ between such a vertex of $\rho(e)$ and such a vertex of $\rho(f)$ that contains no vertices of $C$ and $D$ (except for the end vertices) we say that there is a simple link between the edges $e$ and $f$ of $X(G)$, and we write $e \sim f$. If there is a sequence of simple links

$$
e=e_{0} \sim e_{1}, e_{1} \sim e_{2}, \ldots, e_{r-1} \sim e_{r}=f,
$$

for some $r>0$, we say that $e$ and $f$ are linked.

Our basic strategy is to assume that the set of vertices $W$ is not an articulation set of $G$ and to derive a contradiction. Suppose $W$ is not an articulation set of $G$. Then every edge of $X(G)$ of $H$-length greater than 1 must be linked to every other edge of $X(G)$ of $H$-length greater than 1 . Moreover, there must be a collection of simple links from which these links can be constructed. We use the computer and some simple reasoning to decide which pairs of edges of $X(G)$ can never be simply linked and so to exhibit two edges in $X(G)$ which necessarily must have $H$-length greater than 1 but which are not linked: thus we obtain a contradiction. In more detail, our strategy consists of three phases.

Phase A. Given the graph $X(G)$, we use the computer to try to ascertain whether two given edges $x_{1} y_{1}$ and $x_{2} y_{2}$ are not simply linked, as follows. We first assume that the two edges are indeed simply linked and hope to obtain a contradiction (note that this assumption implies that both of the edges must have $H$-length greater than 1$)$. We augment the graph $X(G)$ with the proposed simple link by (a) removing the edges $x_{1} y_{1}$ and $x_{2} y_{2}$ from $X(G)$; (b) introducing two new vertices $z_{1}$ and $z_{2}$ into $X(G)$ and including the edges $x_{1} z_{1}, z_{1} y_{1}, x_{2} z_{2}$ and 
$z_{2} y_{2}$; and (c) including two edges $z_{1} z_{2}$ in $X(G)$ (recall that $X(G)$ may have multiple edges).

Let the edges $x_{1} y_{1}$ and $x_{2} y_{2}$ correspond to the subpaths $\rho\left(x_{1} y_{1}\right)$ and $\rho\left(x_{2} y_{2}\right)$ of $H(G)$. This augmentation of $X(G)$ corresponds to assuming that there is a path $\rho$ in the graph $G$ from a vertex in $\rho\left(x_{1} y_{1}\right)$ to a vertex in $\rho\left(x_{2} y_{2}\right)$ (where neither of these vertices is a vertex of $W$ ) that has no vertices in common with $C$ and $D$ (the path $\rho$ has been represented twice in $X(G))$.

Note that $X(G)$ now has $k+2$ vertices and $2 k+4$ edges, with each vertex having degree 4 . We now use the computer to check whether there is a Hamiltonian cycle present in $X(G)$ the removal of which leaves another Hamiltonian cycle remaining. If $X(G)$ does consist of two such Hamiltonian cycles, the graph $G$ clearly has two cycles $C^{\prime}$ and $D^{\prime}$ such that every edge of $C$ and $D$ appears in one of $C^{\prime}$ and $D^{\prime}$, and also such that the edges of the path $\rho$ appear in both $C^{\prime}$ and $D^{\prime}$. Hence, the combined lengths of the cycles $C^{\prime}$ and $D^{\prime}$ of $G$ is at least as great as the combined lengths of the cycles $C$ and $D$ plus twice the length of the path $\rho$. This yields a contradiction as $C$ and $D$ are assumed to be longest cycles in $G$ : hence, the edges $x_{1} y_{1}$ and $x_{2} y_{2}$ cannot be simply linked.

Consequently, by following the above procedure for every potential simple link, we end up with a set of simple links from which the set of actual simple links must be drawn. In fact, we do not need to follow the above procedure for every potential simple link as there are some lemmas (to be given in Section 3) which allow us to immediately eliminate some of these simple links: this cuts down on computation time (as discussed in Section 3).

As an example with $k=5$, let $X(G)$ be the graph in Figure 1, left, where $W=\{1,2,3,4,5\}$ and the edges of $X(G)$ are $12,12,14,15,23,23$, $34,35,45$ and 45 .

Suppose we augment $X(G)$ with the potential simple link $23_{Z} \sim 45_{Z}$ (we distinguish between the edges of a parallel pair by adding appropriate subscripts). See Figure 1, right. Then there is a pair of Hamiltonian cycles $(1,2,6,7,5,3,4)$ and
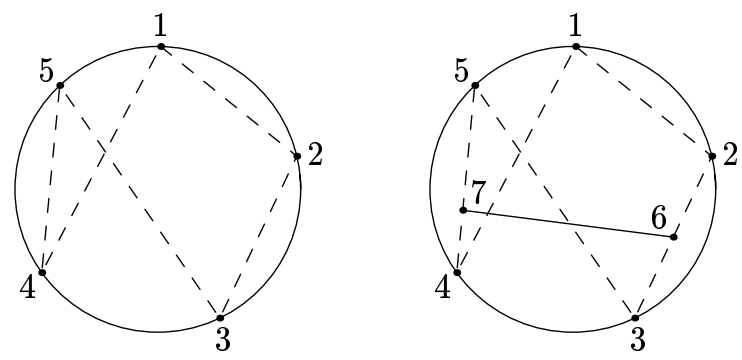

FIGURE 1. The graph $X(G)$ and an augmentation. The edges of the cycle $Y$ are solid, and those of $Z$ are dashed. For ease of readability, we only show one edge 67 , but there are in fact two such edges.

$(1,2,3,6,7,4,5)$, as required; one contains the edge 12 of $Y$, the other the edge 12 of $Z$. Hence, $23_{Z} \sim$ $45_{Z}$ cannot be a simple link. In fact, in this case the computer tells us that the only potential simple links are $12 \sim 34,12 \sim 35,14 \sim 23$ and $15 \sim 23$. (By saying that $12 \sim 34$ is a potential simple link we mean that potentially $12_{Y} \sim 34_{Y}, 12_{Y} \sim 34_{Z}$, $12_{Z} \sim 34_{Y}$ and $12_{Z} \sim 34_{Z}$ are simple links: we adopt this convention throughout.) For this example, one can easily obtain, using the lemmas alluded to above, a contradiction to our initial hypothesis that $W$ is not an articulation set; but we will persevere with this simple example as an aid to our explanations of Phases $\mathrm{B}$ and $\mathrm{C}$ below.

Phase B. Let $S$ be our reduced set of potential simple links. If $e$ is some edge of $X(G)$ that is not involved in any of the simple links in $S$, we may assume that it has $H$-length 1 , for otherwise $W$ is an articulation set of $G$. Also, for each edge $e$ of $X(G)$, we form the set of edges of $X(G)$ reachable from $e$ using the simple links of $S$, and we denote this set by $R(e)$. If $\varnothing \neq R(e) \neq R(f) \neq \varnothing$, for two edges $e$ and $f$ of $X(G)$, we may assume that either all the simple links of $X(G)$ come from those involved in building $R(e)$ or from those involved in building $R(f)$ (otherwise, there will be edges of $H$-length 1 in $R(e)$ and $R(f)$, and these edges can never be linked).

More formally, we form the graph $K$ whose vertices are the edges of $X(G)$ and where there is an edge joining $e$ and $f$, say, if there is a simple link 
$e \sim f$ in $S$. We then find the connected components of this graph $K$ and consider the sets of simple links corresponding to each connected component in turn. The upshot is that we have a number of cases to consider, one for each set of simple links corresponding to some connected component of $K$ (note that these sets of simple links are pairwise disjoint). The point of considering sets of simple links corresponding to the different connected components of $K$ in turn is that we are able to cut down the computation time and also to reduce a more complicated case to at least two simpler subcases (assuming that $K$ has more than one connected component).

Returning to the example of Figure 1, the set of potential simple links is partitioned into sets

$$
\{12 \sim 34,12 \sim 35\} \quad \text { and } \quad\{14 \sim 23,15 \sim 23\}
$$

each set to be considered in turn.

Let $S$ be the set of simple links corresponding to some connected component of $K$. For each simple link in $S$ in turn, we augment $X(G)$ with this simple link, as we did in Phase A above, and enumerate every possible cycle in (the augmented) $X(G)$. For each cycle, we write an expression involving variables corresponding to the $H$-lengths of the edges of this cycle for its total $H$-length, remembering that we could well have knowledge pertaining to which edges of $X(G)$ necessarily have $H$ length 1 . This expression, by definition, must be at most the total $H$-length of the cycle $Y$ and also at most the total $H$-length of the cycle $Z$ of $X(G)$. Hence, for each cycle of $X(G)$, we obtain two inequalities: one saying that the total $H$-length of this cycle is at most the total $H$-length of the cycle $Y$, and one saying that it is at most the total $H$-length of the cycle $Z$. Collecting all these inequalities together, for every cycle in $X(G)$, we obtain a set of inequalities $I$. We then use the computer to decide whether this set of inequalities $I$ has a solution in nonzero natural numbers. If no such solution exists, we can deduce that the simple link with which we augmented $X(G)$ cannot exist.
Hence, after all phases of our analysis, we will be left with some sets of simple links $S_{1}, S_{2}, \ldots, S_{k}$ and the knowledge that the simple links involved in $X(G)$ must come entirely from $S_{1}$ or entirely from $S_{2}$ or $\ldots$ or entirely from $S_{k}$. It turns out that in most cases we can easily then obtain a contradiction to our initial hypothesis that $W$ is not an articulation set.

Returning to our example above, suppose that we augment $X(G)$ with the potential simple link $12_{Z} \sim 34$ as in Figure 2. We may assume that every edge of $X(G)$, with the exception of $12_{Y}, 16$, $26,35,37,47$ and 67 , has $H$-length 1 . The cycle $(1,2,3,5,4,7,6)$ yields the inequalities

$$
\begin{aligned}
& \left|12_{Y}\right|+|35|+|47|+|67|+|16|+2 \leq\left|12_{Y}\right|+|37|+|47|+3, \\
& \left|12_{Y}\right|+|35|+|47|+|67|+|16|+2 \leq|16|+|26|+|35|+3,
\end{aligned}
$$

and the cycle $(1,6,2,3,7,4,5)$ yields

$$
\begin{aligned}
& |16|+|26|+|37|+|47|+3 \leq\left|12_{Y}\right|+|37|+|47|+3, \\
& |16|+|26|+|37|+|47|+3 \leq|16|+|26|+|35|+3 .
\end{aligned}
$$

There are also inequalities $\left|12_{Y}\right| \geq 1,|16| \geq 1$, $|26| \geq 1,|35| \geq 1,|37| \geq 1,|47| \geq 1$, and $|67| \geq$ 1 , as well as similar inequalities for every other cycle of $X(G)$. It is easy to see that the set of 11 inequalities above (and so the full set) does not have a solution; thus there cannot be a simple link $12_{Z} \sim 34$.

We remark that we could omit Phase A of our strategy by simply augmenting $X(G)$ with each potential simple link, in turn, and enumerating all the cycles to obtain a set of inequalities. However, Phase B of our strategy is computationally

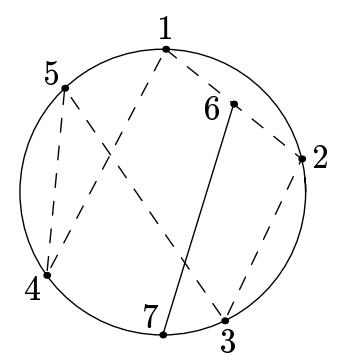

FIGURE 2. The graph $X(G)$, augmented with the simple link $12_{Z} \sim 34$. 
intensive and, in any case, it is preferable to limit Phase B computations to a minimum (see Section 3). Hence, we include Phase A to eliminate unnecessary Phase B computations.

Phase C. As mentioned above, the computation in Phases A and B is enough to settle most cases. However, in order to keep any subsequent reasoning simple, for some cases we prefer to use results from a third phase of computation, Phase C. Recall that after Phases A and B we are left with a set of potential simple links for $X(G)$. We now augment $X(G)$ with a pair of potential simple links simultaneously, as we did in Phase A, and check to see whether the augmented $X(G)$ consists of two disjoint Hamiltonian cycles. This analysis provides us with information as to whether certain pairs of potential simple links can occur simultaneously. However, it is not appropriate to do this for every pair of potential simple links, as we now explain.

By saying that we augment $X(G)$ with two potential simple links simultaneously, we mean that we augment $X(G)$ with the first potential simple link as we did in Phase A, and then we augment the augmented $X(G)$ with the second potential simple link, also as we did in Phase A. Complications might arise, however: firstly, the two potential simple links might share a common edge of $X(G)$; secondly, the two paths in $G$ corresponding to our two potential simple links might not be internally disjoint, so we are not justified in augmenting $X(G)$ as we have described. If our two potential simple links share a common edge of $X(G)$, there are three ways in which to augment $X(G)$ once we have included the first simple link: consequently, to avoid unnecessary complication, we do not apply Phase C computation to any pair of potential simple links that share a common edge of $X(G)$. (Note that this does not make a pair of potential simple links such as $12_{Y} \sim 34$ and $12_{Z} \sim 35$ in the above example exempt from Phase $\mathrm{C}$ computation.)

Also, for the same reason, we only apply Phase C computation to a pair of potential simple links when we can be sure that it cannot be the case that the paths in $G$ corresponding to these two potential simple links are not disjoint.

Continuing still with the example above, suppose that we wanted to augment $X(G)$ with the potential simple links $12_{Y} \sim 34$ and $12_{Z} \sim 35$ simultaneously. We can be sure that any paths in $G$ corresponding to these potential simple links cannot have a vertex in common, for otherwise we would have a simple link $12_{Y} \sim 12_{Z}$, which is forbidden. Hence, $12_{Y} \sim 34$ and $12_{Z} \sim 35$ fall under the remit of Phase $\mathrm{C}$ computation. After the Phase C computation we can easily settle all cases.

Note that our strategy only depends upon the graph $X(G)$ corresponding to some graph $G$ and two longest cycles $C$ and $D$. That is, only a finite number of such graphs $X(G)$ can arise. We begin our analysis by using the computer to generate all nonisomorphic graphs $X(G)$ when $k \in\{6,7\}$, and then we apply our strategy for each $X(G)$ in turn. Doing this yields a proof (albeit computerdependent) of Theorem 1.2. In fact, in many cases we need not apply every phase of the strategy, because a contradiction is often immediate from the computational results of an earlier phase (and possibly some simple reasoning).

\section{REMARKS ON THE COMPUTATIONS}

Given the strategy expounded in the previous section, we have made a conscious decision to keep the programs implementing this strategy as straightforward as possible. Whilst our programs are probably not as elegant or fast as they could be, they are easy to read and understand, and the time taken to achieve our goals is reasonable. Moreover, since we are only concerned with the results of these programs (which in principle won't need to be run again), there is no need for exhaustive optimizations.

The preliminary step of generating the different nonisomorphic cases for $X(G)$ for $k \in\{6,7\}$ is achieved by brute force; for example, a graph $X(G)$ is augmented with a simple link, and whether the resulting graph is a disjoint union of two Hamil- 
tonian cycles is checked by enumerating all Hamiltonian cycles of the graph, removing them in turn and checking to see whether a Hamiltonian cycle remains. This is one of the more computationally intensive parts of the strategy, so we use the following lemmas to eliminate some situations (these lemmas are also used in the subsequent analysis).

Lemma 3.1. Suppose $e$ and $f$ are parallel edges in $X(G)$. Then $e$ and $f$ have the same $H$-length.

Lemma 3.2. Suppose e and $f$ are edges in $X(G)$ with $e$ in the cycle $Y$ and $f$ in the cycle $Z$. If $e$ and $f$ have at least one vertex in common, they are not simply linked.

Lemma 3.3. Suppose e and $f$ are parallel edges in $X(G)$ and $g$ is another edge, distinct from both $e$ and $f$. Then we cannot have both $e \sim g$ and $f \sim g$.

Lemma 3.4. If $P_{1}$ and $P_{2}$ are pairs of parallel edges in $X(G)$, there is at most one simple link between an edge of $P_{1}$ and an edge of $P_{2}$.

Lemmas 3.1, 3.2 and 3.3 are proved in [Grötschel 1984], and Lemma 3.4 can be proved using very similar reasoning. (The results of the computation in Phase A are given in full in Appendix A of [Stewart and Thompson 1994]. Note that we also partition sets of potential simple links into subsets corresponding to connected components of the graph $K$ : see Section 2.)

The computation in Phase B is essentially split into two parts: the enumeration of the cycles of some graph, and the solution of the resulting set of inequalities. The enumeration of cycles is as usual done by brute force, and for checking whether there is a solution to a set of inequalities we use Mathematica [Wolfram 1991]. Whenever this could be accomplished relatively easily, we dispensed with particular cases by using the above lemmas and some simple reasoning. In particular, we tried to limit the use of Mathematica, for two reasons: whilst we may feel confident that code written by us does what it is supposed to, we have no way to guarantee the reliability of a program written in Mathematica; and running Mathematica programs is time- consuming. (The results of the computation in Phases B and C are given in [Stewart and Thompson 1994, Appendices B and C]).

\section{THE DIFFERENT CASES}

Given the method detailed above, it would not be difficult for readers to write the appropriate programs themselves and so prove Theorem 1.2. Consequently, rather than present a complete proof for every case of Theorem 1.2, we highlight here the most difficult cases and leave the others as an exercise. Those readers disinclined to exercise may always consult [Stewart and Thompson 1994], where a complete analysis is given. Henceforth, we do not explicitly refer to Lemmas 3.1-3.4.

For $k=6$, the most problematic case is when $Y=(1,2,3,4,5,6)$ and $Z=(1,2,3,4,6,5)$. The potential simple links remaining after the computation in Phase A are $12 \sim 34,12 \sim 45,12 \sim 46$, $15 \sim 23,15 \sim 34,16 \sim 23,16 \sim 34,23 \sim 45$, $23 \sim 46$, and $23 \sim 56$.

Now suppose $|12|>1$. We must have $12 \sim 34$ and either $12 \sim 45$ or $12 \sim 46$, but not both. If $12 \sim 45$, in order that $12_{Y}$ be linked to $12_{Z}$, we must have $23 \sim 45$. However, according to Phase $\mathrm{C}$ computation, $12 \sim 34$ and $23 \sim 45$ cannot exist simultaneously, which yields a contradiction. We obtain a similar contradiction when $12 \sim 46$. Hence, $|12|=1$ and, by symmetry, $|34|=1$.

Lemma 4.1. Suppose $Y$ and $Z$ are cycles of the form $(1,2, \ldots)$, and let $i, j \notin\{1,2\}$ be such that the edge $1 i$ is not in $Z$ and $2 j$ is not in $Y$. If $|12| \leq 2$, there is no simple link $1 i \sim 2 j$.

Proof. Augment $X(G)$ with the simple link $1 i \sim 2 j$, as we did in Phase A, from a vertex 8 on $1 i$ to a vertex 9 on $2 j$. Compare the $H$-length of $Y$ with that of the cycle obtained from $Y$ by replacing the edge 12 with the edges 18,89 and 29: this yields a contradiction. The other case is similar.

By Lemma 4.1 , we have $15 \nsim 23,16 \nsim 23,23 \nsim 45$ and $23 \nsim 46$. Hence, $|23|=1$, which yields a contradiction. 
For $k=7$, the most problematic case is when

$Y=(1,2,3,4,5,6,7)$ and $Z=(1,2,3,4,5,7,6)$.

The potential simple links remaining after Phase A computation are $12 \sim 35,12 \sim 45,12 \sim 56$, $12 \sim 57,16 \sim 23,16 \sim 24,16 \sim 34,16 \sim 35$, $16 \sim 45,17 \sim 23,17 \sim 24,17 \sim 34,17 \sim 35$, $17 \sim 45,23 \sim 56,23 \sim 57,23 \sim 67,24 \sim 56$, $24 \sim 57,24 \sim 67$.

If $|34|>1$, without loss of generality $16 \sim 34_{Y}$ and $17 \sim 34_{Z}$ : but these two simple links cannot exist simultaneously and so $|34|=1$. Similarly, $|67|=1$.

Suppose that $|12|>1$ and that $12_{Y} \sim 35$ and $12_{Z} \sim 56$ : note that no other simple links involve 12 . In order that $12_{Y}$ be linked to $12_{Z}$, it must be the case that 56 is involved in some other simple link; but the Phase $\mathrm{C}$ computation tells us that this is impossible and so it cannot be the case that $12_{Y} \sim 35$ and $12_{Z} \sim 56$. By proceeding similarly, no matter which two simple links involving 12 we choose, we always obtain a contradiction: thus $|12|=1$.

By Lemma 4.1, $16 \nsim 24$ and $17 \nsim 23$. Also, by proceeding as we did in the proof of Lemma 4.1, $16 \nsim 23$ and $17 \nsim 24$. Hence, the set of simple links must be a subset of either of the two sets

$$
\begin{aligned}
& \{16 \sim 35,16 \sim 45,17 \sim 35,17 \sim 45\}, \\
& \{23 \sim 56,23 \sim 57,24 \sim 56,24 \sim 57\} .
\end{aligned}
$$

Without loss of generality we may suppose that it is a subset of the first of these sets. By Lemma 4.1,
$16 \nsim 35$ and $17 \nsim 45$, and the Phase C computation tells us that $16 \sim 45$ and $17 \sim 35$ cannot occur simultaneously. If $16 \sim 45$, comparing the $H$-length of the cycle $(1,6,7,5,4,3,2)$ with that of $Y$ yields that $|16|=1$, from which we obtain a contradiction. The case when $17 \sim 35$ is similar.

\section{REFERENCES}

[Babai 1979] L. Babai, "Long cycles in vertex-transitive graphs", J. Graph Theory 3 (1979), 301-304.

[Grötschel 1984] M. Grötschel, "On intersections of longest cycles", pp. 171-189 in Graph Theory and Combinatorics: Proceedings of the Cambridge Combinatorial Conference in Honour of Paul Erdös (edited by B. Bollobás), Academic Press, London, 1984.

[Mader 1971] W. Mader, "Eine Eigenschaft der Atome endlicher Graphen", Arch. Math. (Basel) 22 (1971), $333-336$.

[Stewart and Thompson 1994] I. A. Stewart and B. C. Thompson, "On the intersections of longest cycles in a graph", Technical Report CSR 14-94, University of Wales Swansea, 1994.

[Watkins 1970] M. E. Watkins, "Connectivity of transitive graphs", J. Combinat. Theory 8 (1970), 23-29.

[Wolfram 1991] S. Wolfram, Mathematica: A System for Doing Mathematics by Computer, 2nd ed., Addison-Wesley, Reading, MA, 1991.

\section{ELECTRONIC AVAILABILITY}

Our programs are available by anonymous ftp from the host info.swan.ac.uk, in directory /pub/compsci/cycles. See also [Stewart and Thompson 1994].

Iain A. Stewart, Department of Computer Science, University of Wales Swansea, Swansea SA2 8PP, U.K.

(I.A.Stewart@swansea.ac.uk)

Ben Thompson, Department of Computer Science, University of Wales Swansea, Swansea SA2 8PP, U.K.

(B.C.Thompson@swansea.ac.uk) 